\title{
Momentum transfer squared dependence of exclusive quarkonia photoproduction in ultraperipheral collisions
}

\author{
Cheryl Henkels $\odot,{ }^{1, *}$ Emmanuel G. de Oliveira ${ }^{1, \dagger}$ Roman Pasechnik $\odot,{ }^{1,2, \ddagger}$ and Haimon Trebien $\circledast^{1, \S}$ \\ ${ }^{1}$ Departamento de Física, CFM, Universidade Federal de Santa Catarina, \\ C.P. 476, CEP 88.040-900 Florianópolis, Santa Catarina, Brazil \\ ${ }^{2}$ Department of Astronomy and Theoretical Physics, Lund University, SE-223 62 Lund, Sweden
}

(Received 19 April 2021; accepted 28 July 2021; published 9 September 2021)

\begin{abstract}
We study fully differential quarkonia photoproduction observables in ultraperipheral collisions as functions of momentum transfer squared. We employ the dipole picture of the QCD part of the scattering with proton and nucleus targets, with the projectile being a quasireal photon flux emitted by an incoming hadron. We analyze such observables for ground $J / \psi, \Upsilon(1 S)$ and excited $\psi^{\prime}, \Upsilon(2 S)$ states whose lightfront wave functions are obtained in the framework of an interquark potential model incorporating the Melosh spin transformation. Two different low- $x$ saturation models, one obtained by solving the BalitskyKovchegov equation with the collinearly improved kernel and the other with a Gaussian impact-parameterdependent profile, are used to estimate the underlined theoretical uncertainties of our calculations. The results for the proton target and with charmonium in the final state are in agreement with the available HERA data, while in the case of the nucleus target we make predictions for $\gamma A$ and $A A$ differential cross sections at different $W$ and at $\sqrt{s}=5.02 \mathrm{TeV}$, respectively.
\end{abstract}

DOI: $10.1103 /$ PhysRevD.104.054008

\section{INTRODUCTION}

The determination of the structure of protons and nuclei in terms of their fundamental constituents as well as their interactions is one of the biggest goals of particle physics [1]. An important milestone for the proton structure measurements was the start of operation of the HERA collider at DESY. There, a large amount of deep inelastic scattering (DIS) data (in which simple pointlike leptons are used to probe the proton substructure) has been collected, making it possible to extract a detailed knowledge about the parton distribution functions (PDFs) for the proton with good precision for as low a longitudinal momentum fraction $x$ as $10^{-5}$ or so [2].

To obtain a more detailed picture of the target, in particular, to access information about its transverse shape at a given $x$, more differential observables are needed. Two processes that provide such observables, deeply virtual Compton scattering (DVCS) (where the outgoing photon is

\footnotetext{
*cherylhenkels@hotmail.com

†emmanuel.de.oliveira@ufsc.br

Roman.Pasechnik@thep.lu.se

\$haimontrebien@outlook.com
}

Published by the American Physical Society under the terms of the Creative Commons Attribution 4.0 International license. Further distribution of this work must maintain attribution to the author(s) and the published article's title, journal citation, and DOI. Funded by SCOAP. real) and the exclusive production of vector mesons (with the same quantum numbers $J^{P C}=1^{--}$as those of the photon), are frequently discussed in the literature. In the first case, thanks to the high beam energy available at the HERA collider, the experiments $\mathrm{H} 1$ and ZEUS have measured the pure DVCS cross section for a Bjorken variable ranging from $10^{-4}$ to $10^{-2}$. In the second case, besides exclusive electro- and photoproduction of light vector mesons $(\phi, \rho)$ and quarkonia $(J / \psi)$ studied by the $\mathrm{H} 1$ and ZEUS collaborations, there are more recent data on vector-meson photoproduction in ultraperipheral collisions (UPCs) available from the LHC. The latter processes are the main focus of this work.

Particle production processes in proton-nucleus $p A$ and nucleus-nucleus $A A$ UPCs have attracted a lot of attention in recent years due to their vast potential in probing the proton and nucleus structure at very small $x$ (for a recent review, see, e.g., Ref. [3]). A particularly clean environment in UPCs is achieved in a fully exclusive process when a small-mass hadronic system is produced that is separated from the intact scattered particles by large rapidity gaps on both sides. A phenomenologically important and wellknown example of such a scattering refers to exclusive quarkonia [such as charmonia $J / \psi \equiv \psi(1 S), \psi^{\prime} \equiv \psi(2 S)$, and bottomonia $\Upsilon(1 S, 2 S)$ ] photoproduction reactions in UPCs that have recently gained a particular relevance motivated by a wealth of experimental data coming from the LHC, such as those from LHCb [4-6], ALICE [7-12], and CMS [13,14] experiments. 
The process is straightforwardly visualized by considering it in the target rest frame. While on one side of the collision a photon flux is emitted from a fast projectile (hadron or nucleus) and then fluctuates into a color-neutral $Q \bar{Q}(Q=c, b)$ pair called a color dipole, on another side such a dipole coherently rescatters off the target by means of an exchange of a multiple gluonic system in a colorsinglet state-a dominating configuration at low longitudinal momentum transfers, $x$. In the leading-order perturbative quantum chromodynamics (QCD) approximation, typically validated by having a hard scale associated with the heavy-quark mass $m_{Q}$, one considers a colorless gluonpair exchange between the dipole and the target. In the limit of small $x \ll 1$ and low four-momentum transfer squared $|t|=-\left(p_{1}-p_{1}^{\prime}\right)^{2} \ll m_{Q}^{2}$, such an exchange in momentum space is usually described in terms of the generalized unintegrated gluon density in the target, which, in turn, connects to the dipole scattering matrix as a function of gluon $x$, dipole separation $\vec{r}$, and the impact parameter of the scattering $\vec{b}$. This matrix effectively encodes dynamics of parton saturation and contains full information about the relative dipole orientation with respect to the color background field of the target. As long as $\vec{r}$ is integrated out in a convolution with the quarkonium light-front (LF) wave function, the impact-parameter dependence provides the transverse profile of the target gluon density that can be probed by means of the measured differential in $t$ distributions.

The impact-parameter dependence of the gluon density in the target is an intrinsically nonperturbative property and is often parametrized in terms of a Gaussian distribution, as is done, for example, in the case of the so-called bSat model [15]. To get a more accurate description of interactions between the color dipole and the target encoded in the impact-parameter profile of the target, the corresponding amplitude can be found by solving the Balitsky-Kovchegov (BK) evolution equation [16,17]. It is known that the BK equation at next-to-leading order (NLO) is unstable due to large NLO corrections when one integrates out the gluon emissions with small transverse momenta. Thus, these corrections need to be properly resummed to all orders [18]. An additional phenomenon called Coulomb tails that corresponds to an unphysical growth of the amplitude at large impact parameters should be taken into consideration. The latter phenomenon is found to be connected to the creation of large daughter dipoles during the evolution, thus enabling this problem to be cured. The BK solutions without such Coulomb tails can be found in several recent studies; e.g., in Refs. $[19,20]$ this problem is absent owing to the use of a collinearly improved kernel. In the current analysis, we apply both the bSat model and the BK solution with a collinearly improved kernel in the study of differential quarkonia photoproduction cross sections in UPCs for relevant experimental conditions at HERA and LHC colliders.
The paper is organized as follows. In Sec. II, we give a short description of the differential cross section of elastic vector-meson photoproduction $\gamma p \rightarrow V p$ off the proton target in terms of the dipole $S$-matrix and quarkonia LF wave functions in the framework of potential approach. In Sec. III, we discuss the models for the impact-parameterdependent partial dipole amplitude that have been used in the numerical analysis throughout this work. Section IV presents the numerical results for the differential cross section of the $\gamma p \rightarrow V p$ process for the ground and excited quarkonia states, with $J / \psi$ results successfully describing the existing data. In Sec. V, we review the formalism to obtain the differential cross section of coherent quarkonia photoproduction off nuclear targets in UPCs and show our corresponding numerical predictions for the ground and first excited $\psi$ and $\Upsilon$ states presented in Sec. VI. Finally, a brief summary of our results is given in Sec. VII.

\section{ELASTIC PHOTOPRODUCTION OFF A PROTON}

The advantage of studying vector-meson photoproduction is that, in order to produce a single vector meson and nothing else in a detector, a color charge cannot be transferred to the target, requiring that at least two gluons (in the net color-singlet state) are exchanged. This provides an exclusive character for the process, with a particularly clean environment. Another advantage is that only in the exclusive scattering process is it possible to measure the total momentum transfer $\Delta_{T}$ and interpret it as the Fourier conjugate of the impact parameter (see, e.g., Refs. [21,22]). Consequently, these processes probe not only the density of partons but also their spatial distribution in the transverse plane.

Considering the proton target case first, at high energies the elastic diffractive differential cross section for the $\gamma p \rightarrow$ $V p$ scattering is found as follows [15]:

$$
\frac{\mathrm{d} \sigma^{\gamma p \rightarrow V p}}{\mathrm{~d} t}=\frac{1}{16 \pi}\left|\mathcal{A}^{\gamma p}\left(x, \Delta_{T}\right)\right|^{2},
$$

where $t=-\Delta_{T}^{2} \equiv\left(p_{1}-p_{1}^{\prime}\right)^{2}$ is the momentum transfer squared, $\Delta_{T} \equiv|\boldsymbol{\Delta}|$ is the transverse momentum of the produced vector meson $V$ recoiled against the target (assuming the projectile photon momentum to be collinear, i.e., that it carries no transverse momentum) and the elastic production amplitude

$$
\mathcal{A}^{\gamma p}\left(x, \Delta_{T}\right)=\int \mathrm{d}^{2} \boldsymbol{r} \int_{0}^{1} \mathrm{~d} z\left(\Psi_{V}^{*} \Psi_{\gamma}\right) \mathcal{A}_{q \bar{q}}(x, \boldsymbol{r}, \boldsymbol{\Delta})
$$

is given in terms of the overlap between the transversely polarized real photon $\gamma \rightarrow Q \bar{Q}\left(\Psi_{\gamma}\right)$ and vector meson $V \rightarrow$ $Q \bar{Q} \mathrm{LF}$ wave functions ( $\Psi_{\gamma}$ and $\Psi_{V}$, respectively). Here, the elementary amplitude for elastic $q \bar{q}$ dipole scattering $\mathcal{A}_{q \bar{q}}$ is related to the dipole $S$ matrix 


$$
\begin{aligned}
\mathcal{A}_{q \bar{q}}(x, \boldsymbol{r}, \boldsymbol{\Delta}) & =\int \mathrm{d}^{2} \boldsymbol{b} \mathrm{e}^{-\mathrm{i} \boldsymbol{b} \cdot \boldsymbol{\Delta}} \mathcal{A}_{q \bar{q}}(x, \boldsymbol{r}, \boldsymbol{b}) \\
& =\mathrm{i} \int \mathrm{d}^{2} \boldsymbol{b} \mathrm{e}^{-\mathrm{i} \boldsymbol{b} \cdot \boldsymbol{\Delta}} 2[1-S(x, \boldsymbol{r}, \boldsymbol{b})],
\end{aligned}
$$

and thus contains the most detailed (five-dimensional) information about the gluon density in the target. It is directly connected to the so-called gluon Wigner distribution, as was established previously in Ref. [23]. Even though direct access of the elliptic gluon density in the Wigner distribution by a measurement of the exclusive quarkonia photoproduction is impossible due to an $\boldsymbol{r}$ variable being integrated into the measured differential cross section, access to the impact-parameter profile of the target gluon density is still very relevant for understanding the hadron or nucleus structure at very low momentum transfers.

Note that, by means of the optical theorem, the imaginary part of the partial dipole amplitude in the forward limit $\left(\Delta_{T} \rightarrow 0\right)$ is related to the dipole cross section $\sigma_{q \bar{q}}(x, r)$ : a universal ingredient whose parametrization can be extracted from a given process (typically from DIS) and then used for the description of many other processes in $e p, p p$, and $p A$ collisions [24,25] (for early analyses of elastic charmonia photoproduction in the dipole picture, see, e.g., Refs. [26-30]).

In the off-forward case, one straightforwardly rewrites the elastic amplitude in terms of the imaginary part of the elastic $q \bar{q}$ amplitude in the impact-parameter representation in the following way [15]:

$$
\begin{aligned}
\mathcal{A}^{\gamma p}\left(x, \Delta_{T}\right)= & 2 i \int \mathrm{d}^{2} \boldsymbol{r} \int_{0}^{1} \mathrm{~d} z \\
& \times \int \mathrm{d}^{2} \boldsymbol{b}\left(\Psi_{V}^{*} \Psi\right) \mathrm{e}^{-i[\boldsymbol{b}-(1-z) \boldsymbol{r}] \cdot \boldsymbol{\Delta}} N(x, \boldsymbol{r}, \boldsymbol{b}),
\end{aligned}
$$

where $z$ is the longitudinal momentum fraction of a heavy (anti)quark in the $Q \bar{Q}$ dipole and

$N(x, \boldsymbol{r}, \boldsymbol{b}) \equiv \operatorname{Im} \mathcal{A}_{q \bar{q}}(x, \boldsymbol{r}, \boldsymbol{b})=2[1-\operatorname{Re} S(x, \boldsymbol{r}, \boldsymbol{b})]$,

such that the dipole cross section is defined as follows:

$$
\sigma_{q \bar{q}}(x, r)=2 \int \mathrm{d}^{2} \boldsymbol{b} N(x, \boldsymbol{r}, \boldsymbol{b}) .
$$

To take into account the real part of the $\mathcal{A}_{q \bar{q}}$ amplitude, it suffices to introduce into Eq. (2.1) a factor that represents the ratio of the real to imaginary parts of the exclusive photoproduction amplitude $\mathcal{A}^{\gamma p}$ as follows [31]:

$\mathcal{A}^{\gamma p} \Rightarrow \mathcal{A}^{\gamma p}\left(1-i \frac{\pi \lambda}{2}\right), \quad$ with $\quad \lambda=\frac{\partial \ln \mathcal{A}^{\gamma p}}{\partial \ln (1 / x)}$.

At last, one typically also incorporates the so-called skewness effect of the off-diagonal gluon distribution, which takes into account the fact that the gluons exchanged between the $q \bar{q}$ pair and the target can carry very different fractions of the target's momentum $\left(x\right.$ and $\left.x^{\prime}\right)$, while in dipole cross section parametrizations fitted to inclusive DIS data they appear to be the same due to the optical theorem. Thus, considering the dominant kinematical configuration with $x^{\prime} \ll x \ll 1$, the skewness effect is typically included via a multiplicative factor $R_{g}^{2}$ applied to the differential cross section in Eq. (2.1) (see, e.g., Ref. [32]), with

$$
R_{g}(\lambda)=\frac{2^{2 \lambda+3}}{\sqrt{\pi}} \frac{\Gamma(\lambda+5 / 2)}{\Gamma(\lambda+4)},
$$

where $\lambda$ is taken from Eq. (2.7).

Following our previous work [33], we have used the vectormeson wave functions calculated within the potential approach, which relies on factorization of the wave function into the spin-dependent and radial components. In the rest frame of the color dipole, the radial wave function is found to be a numerical solution of the Schrödingerequation, which can be solved for different models for the interquark potential and then boosted to the infinite momentum frame, where the dipole formula for the vector-meson production amplitude (2.4) is defined. In this analysis, we use five different models for the $Q \bar{Q}(Q=c, b)$ interaction potential: powerlike model (pow) [34,35], harmonic oscillator (osc), Cornell potential (cor) [36,37], Buchmüller-Tye parametrization (but) [38], and logarithmic potential $(\log )$ [39]. These models have been fitted to the hadron spectrum and, when solving the Schrödinger equation, we have used the same parameters as in the original fits, including the heavy-quark masses.

However, it is worth mentioning that the quark masses obtained by fitting the interquark potential are not bare masses, as they carry nonperturbative effects that are different for every potential and they are allowed to vary in order to produce a better parametrization of the potential. Thus, in order to maintain the universality of the color dipole model, we chose to use, in the short-distance amplitudes, fixed perturbative masses given by $m_{c}=1.4 \mathrm{GeV}$ and $m_{b}=$ $4.75 \mathrm{GeV}$ for charm and bottom quarks, respectively.

When performing the Lorentz transformation between the two frames, not only should the radial part be properly boosted, but also the spin-dependent part has to be transformed accordingly. Such a transformation is known as the Melosh spin rotation of the quark spinors [40], which causes an important impact on the differential photoproduction cross section, especially for excited quarkonia states [41,42] (for a detailed analysis of the Melosh spin rotation effect, see Ref. [31]). Indeed, the spin rotation increases the groundstate quarkonia cross sections by approximately $30 \%$, while for the excited states the increase is by a factor of 2 to 3 , thereby playing an important role in the description of the exclusive vector-meson photoproduction data.

Using such a quarkonium wave function in Eq. (2.4), the resulting photoproduction amplitude (considering the transversely polarized real photon only) is given by 


$$
\mathcal{A}_{T, L}^{\gamma p}\left(x, \Delta_{T}\right)=2 i \int \mathrm{d}^{2} \boldsymbol{r} \int_{0}^{1} \mathrm{~d} z \int \mathrm{d}^{2} \boldsymbol{b} \mathrm{e}^{-i[\boldsymbol{b}-(1-z) \boldsymbol{r}] \cdot \boldsymbol{\Delta}}\left[\Sigma^{(1)}(z, r) N(x, \boldsymbol{r}, \boldsymbol{b})+\Sigma^{(2)}(z, r) N_{r}^{\prime}(x, \boldsymbol{r}, \boldsymbol{b})\right]
$$

where $N_{r}^{\prime} \equiv d N / d r$,

$$
\Sigma^{(1)}=\frac{Z_{Q} \sqrt{N_{c} \alpha_{\mathrm{em}}}}{2 \pi \sqrt{2}} 2 K_{0}\left(m_{Q} r\right) \int \mathrm{d} p_{T} J_{0}\left(p_{T} r\right) \Psi_{V}\left(z, p_{T}\right) p_{T} \frac{m_{T} m_{L}+m_{T}^{2}-2 z(1-z) p_{T}^{2}}{m_{L}+m_{T}},
$$

and

$$
\Sigma^{(2)}=\frac{Z_{Q} \sqrt{N_{c} \alpha_{\mathrm{em}}}}{2 \pi \sqrt{2}} 2 K_{0}\left(m_{Q} r\right) \int \mathrm{d} p_{T} J_{1}\left(p_{T} r\right) \Psi_{V}\left(z, p_{T}\right) \frac{p_{T}^{2}}{2} \frac{m_{L}+m_{T}+(1-2 z)^{2} m_{T}}{m_{T}\left(m_{L}+m_{T}\right)}
$$

Here, $\alpha_{\mathrm{em}}=1 / 137$ is the fine structure constant, $N_{c}=3$ is the number of colors in QCD, $Z_{Q}$ and $m_{Q}$ are the electric charge and the mass of the heavy quark, respectively, $J_{0,1}$ $\left(K_{0}\right)$ is the Bessel (modified Bessel) function of the first (second) kind, respectively, $p_{T}$ is the transverse momentum of the produced quarkonium state, and

$$
m_{T}=\sqrt{m_{Q}^{2}+p_{T}^{2}}, \quad m_{L}=2 m_{Q} \sqrt{z(1-z)}
$$

It is worth mentioning that there are still significant theoretical uncertainties in the description of the vectormeson wave functions. In addition to the approach discussed above, there are other attempts to model the uncertainties. A very recent one [43] executes the calculations at the NLO level in $\gamma p$ collisions for longitudinally polarized photons making use of the color glass condensate framework and proposing a wave function based upon nonrelativistic QCD matrix elements [44]. Another study [45] modifies the dipole cross section to enhance the suppression of dipoles with large separations beyond the confinement length scale (a correction important for small $Q^{2}$ ). The analysis of Ref. [46] is very similar to ours except that the boosted Gaussian has been utilized there to construct the vector-meson wave functions.

\section{PARTIAL DIPOLE AMPLITUDE}

For the main purpose of scanning of the impact-parameter profile of the target nucleon or nucleus, we need an impact-parameter-dependent (or b-unintegrated) dipole cross section that can be found in terms of the dipole $S$ matrix introduced in Eq. (2.3). We first tested seven different models available in the literature, and we then selected the two that best describe the exclusive vectormeson photoproduction data from the HERA collider, namely, the impact-parameter dipole saturation model [15] (dubbed bSat in what follows) and the model based upon a numerical solution of the BK equation [19].

In the first case of bSat, we employ the following formula:
$N(x, \boldsymbol{r}, \boldsymbol{b})=1-\exp \left(-\frac{\pi^{2}}{2 N_{c}} r^{2} \alpha_{s}\left(\mu^{2}\right) x g\left(x, \mu^{2}\right) T(b)\right)$,

where $\mu^{2}=4 / r^{2}+\mu_{0}^{2}$ is the momentum scale in the collinear gluon density $x g\left(x, \mu^{2}\right)$ and no nontrivial information about the relative dipole orientation is implemented. In numerical calculations, we have used the CT14LO parametrization [47] inspired by our previous analysis of integrated quarkonia photoproduction cross sections performed in Ref. [33]. This will be different from the original bSat model, in which the gluon PDF is evolved up to the scale $\mu^{2}$ with LO Dokshitzer-Gribov-Lipatov-AltarelliParisi gluon evolution neglecting its coupling to quarks, but the numerical results will be similar enough to neglect the difference. In addition, we considered a conventional Gaussian form for the proton shape function $T(b)$,

$$
T(b)=\frac{1}{2 \pi B_{\mathrm{G}}} \mathrm{e}^{-b^{2} / 2 B_{\mathrm{G}}},
$$

where the slope parameter $B_{\mathrm{G}}=4.25 \mathrm{GeV}^{-2}$ is taken from Ref. [48].

In the second case, the numerical solution of the BK equation is provided by Ref. [19], where it is obtained under the assumption that the dipole partial amplitude depends only on the absolute values of the transverse separation of the dipole $r$ and the impact parameter $b$, not on the angle between $\boldsymbol{r}$ and $\boldsymbol{b}$ as in the bSat model. In this case, the BK equation reads

$$
\begin{aligned}
\frac{\partial \mathcal{N}(r, b, Y)}{\partial Y}= & \int d^{2} \boldsymbol{r}_{1} K\left(r, r_{1}, r_{2}\right)\left(\mathcal{N}\left(r_{1}, b_{1}, Y\right)\right. \\
& +\mathcal{N}\left(r_{2}, b_{2}, Y\right)-\mathcal{N}(r, b, Y) \\
& \left.-\mathcal{N}\left(r_{1}, b_{1}, Y\right) \mathcal{N}\left(r_{2}, b_{2}, Y\right)\right),
\end{aligned}
$$

whose numerical solution provides us with the partial dipole amplitude

$$
N(x, \boldsymbol{r}, \boldsymbol{b})=\mathcal{N}(r, b, \ln (0.008 / x))
$$



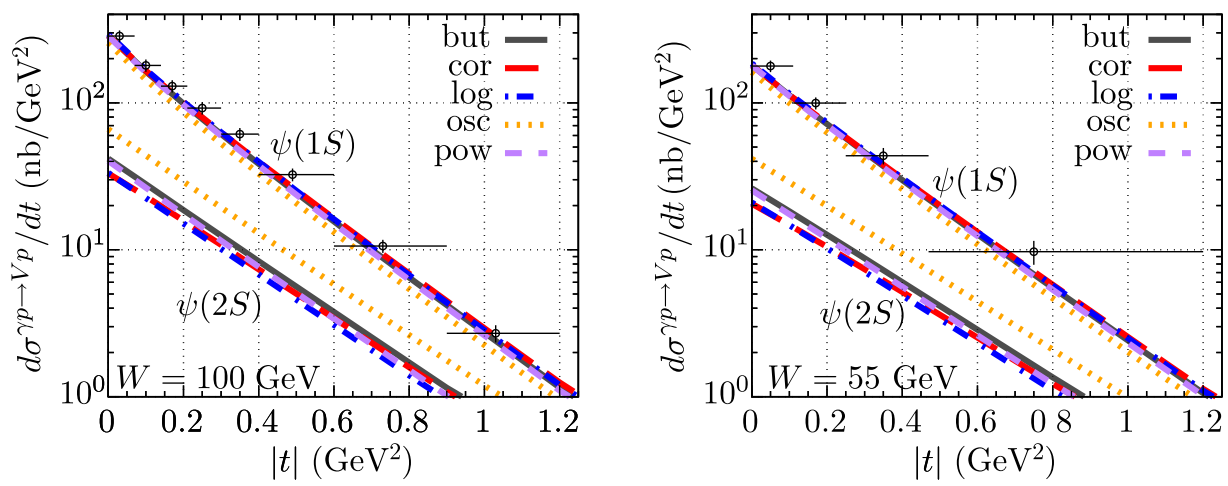

FIG. 1. Differential cross section for $\psi(1 S)$ (upper curves) and $\psi(2 S)$ (lower curves) photoproduction as a function of $|t|$ obtained using the numerical solution of the BK equation obtained in Ref. [19], for $W=100 \mathrm{GeV}$ (left panel) and $W=55 \mathrm{GeV}$ (right panel). The results are presented for five different interquark potential models. The $\psi(1 S)$ results are compared to the corresponding data from the H1 Collaboration [53,54].

which is employed in our numerical analysis below. The specific main feature of the Ref. [19] solution is that it is obtained with a collinearly improved kernel $K\left(r, r_{1}, r_{2}\right)$ studied in Ref. [49] that suppresses the larger daughter dipole sizes during the evolution and thus does not show the nonphysical Coulomb tails.

Finally, following Refs. [50-52], we also incorporate a correction relevant at large $x$ multiplying the dipole cross section by a factor $(1-x)^{2 n_{s}-1}$, where $n_{s}$ denotes the number of spectator quarks, which was chosen to be $n_{s}=4$.

\section{RESULTS FOR $\gamma p \rightarrow V p$ PROCESS}

Now that we have outlined the basic dipole formalism needed for analysis of the differential photoproduction observables, let us first present the numerical results for the $\gamma p \rightarrow V p$ process. Note that, in general, the differential photoproduction cross sections computed for the proton target are very sensitive to the dipole parametrization used in the analysis. In this work, we analyzed many different $b$ dependent parametrizations for the partial dipole amplitude, and they give very different results. We chose to present only those results obtained with the BK solution and the bSat model briefly described above as those that provide the best description of the available $J / \psi$ data. We will start with the BK solution model.

Figure 1 shows the differential cross section for $J / \psi \equiv$ $\psi(1 S)$ (upper curves) and $\psi(2 S)$ (lower curves) production as a function of the momentum transfer squared $|t|$ for $W=$ $100 \mathrm{GeV}$ (left panel) and $W=55 \mathrm{GeV}$ (right panel). Here, the results are obtained using a numerical solution of the BK equation of the $b$-dependent partial dipole amplitude discussed above. The ground-state charmonium results were compared to the experimental data available from the H1 Collaboration [53,54], yielding a very good description. The corresponding observables have been evaluated with the LF quarkonia wave functions obtained for several different parametrizations of the interquark $Q \bar{Q}$ potential (for more details, see Refs. [33,42]), which leads to a rather minor variation in the final results. A bigger difference is found for the $\psi(2 S)$ cross section computed with the harmonic oscillator potential, which is noticeably higher than the results for other potentials. This effect is due to a specific shape of this wave function, as was briefly discussed in Ref. [33]. The $|t|$ slope is close to a constant due to an almost exponential impact-parameter profile of the partial dipole amplitude, in full consistency with the $J / \psi$ data. One notices, however, a somewhat larger difference in the slopes of $J / \psi$ and $\psi(2 S)$ differential cross sections due to different shapes of the wave functions.

In Fig. 2 we present our predictions for the differential cross section of $\Upsilon(1 S)$ (left panel) and $\Upsilon(2 S)$ (right panel) photoproduction as a function of $|t|$, also using the numerical solution of the BK equation, for $W=120 \mathrm{GeV}$. The results for the ground and excited states are separated into two different plots since the corresponding results for the oscillator potential are very close. This occurs due to the fact that these two wave functions in the case of a harmonic oscillator have a very similar small- $r$ dependence. Since this domain plays a dominant role in the integration of the $\Upsilon$ production amplitudes, one indeed arrives at very similar numerical results for $\Upsilon(1 S)$ and $\Upsilon(2 S)$ photoproduction in this case.

Figures 3 and 4 represent the quantities shown in Figs. 1 and 2, respectively, except that the former are computed with the bSat dipole parametrization instead of the BK solution employed in the latter. As can be seen in Fig. 3, the use of the bSat dipole model and the LF quarkonia wave functions calculated within the potential approach also provides a fair description of the H1 data. The latter is not as good as that found in the case of the BK solution, though. However, since bSat dipole parametrization is widely used in the literature, in this work we chose to show the corresponding numerical results as well. A comparison between the curves obtained 

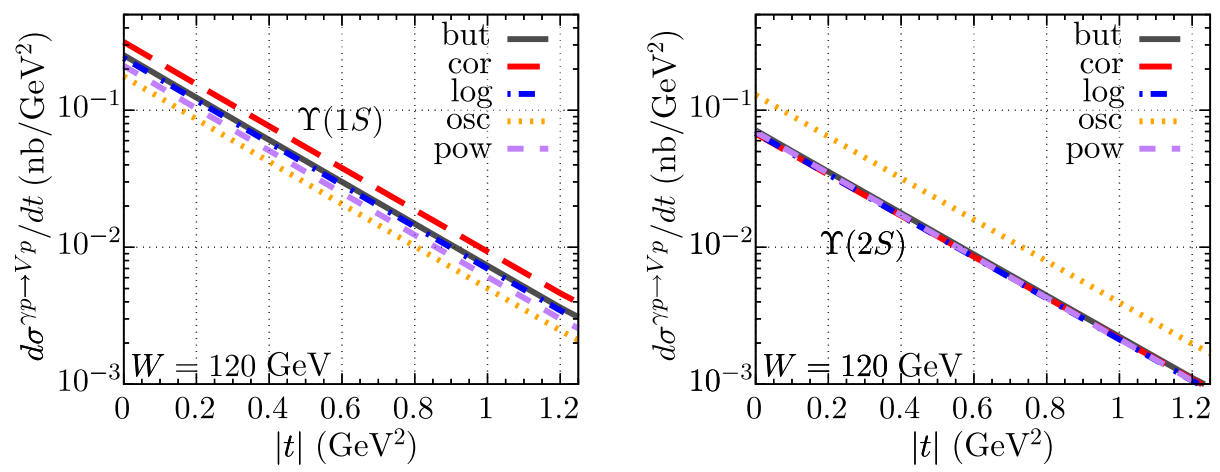

FIG. 2. Predictions for the differential cross section for $\Upsilon(1 S)$ (left panel) and $\Upsilon(2 S)$ (right panel) as a function of $|t|$ obtained using the numerical solution of the BK equation obtained in Ref. [19] for $W=120 \mathrm{GeV}$. The results are presented for five different interquark potential models.
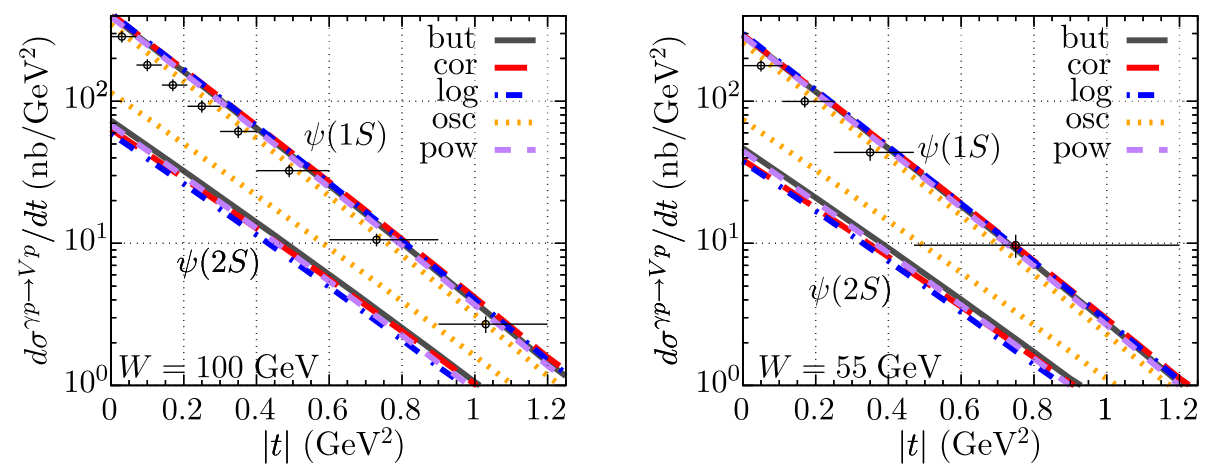

FIG. 3. Differential cross section for $\psi(1 S)$ (upper curves) and $\psi(2 S)$ (lower curves) photoproduction as a function of $|t|$ found with the bSat dipole model for $W=100 \mathrm{GeV}$ (left panel) and $W=55 \mathrm{GeV}$ (right panel) including the skewness effect. The results are presented for five different interquark potential models. The $\psi(1 S)$ results are compared to the corresponding data from the H1 Collaboration [53,54].
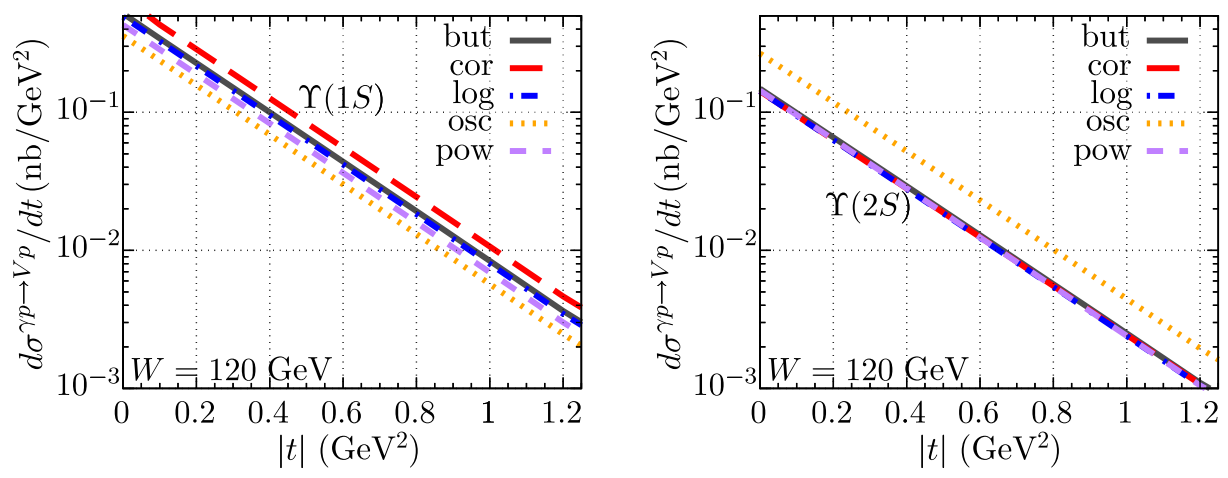

FIG. 4. Predictions for the differential cross section for $\Upsilon(1 S)$ (left panel) and $\Upsilon(2 S)$ (right panel) photoproduction as a function of $|t|$ computed with the bSat dipole model for $W=120 \mathrm{GeV}$. The results are presented for five different interquark potential models.

with these two dipole models and the available H1 data for $\psi(1 S)$ photoproduction is presented in Fig. 5, where we can see that both curves found are located mainly within the experimental error bars for both $W=100 \mathrm{GeV}$ (left panel) and $W=55 \mathrm{GeV}$ (right panel), except that at small $|t|$ and large $W$ the bSat model marginally overshoots the data.
Finally, we include Fig. 6, which shows our results on the photoproduction cross section of $\Upsilon$ states in $p P b$ collisions when the photon is emitted from the nucleus. The required photon flux will be discussed in the next section. These results are compared with the CMS data points [14]. In the plot the curve is obtained by summing each $\Upsilon(n S)$ state 

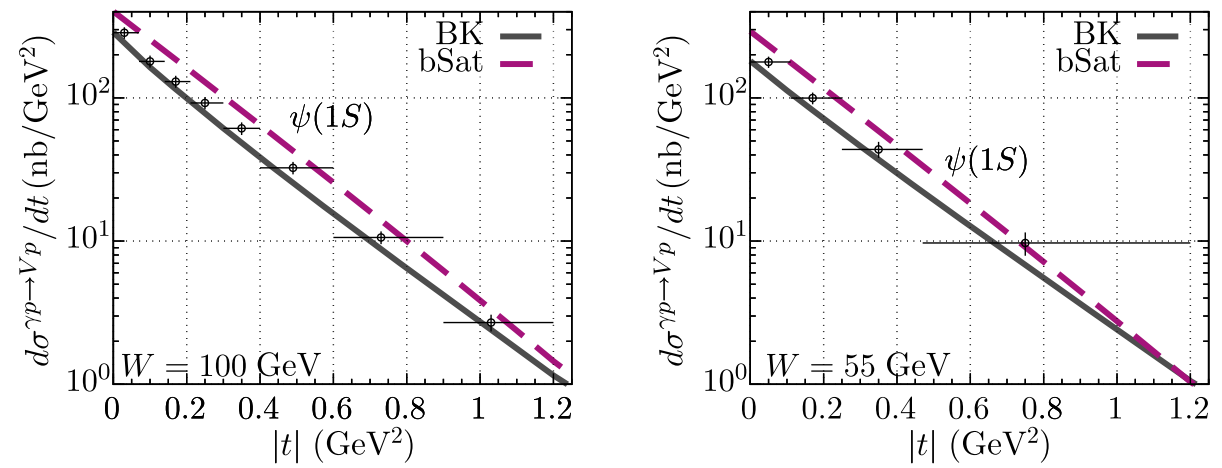

FIG. 5. Differential cross section for $\psi(1 S)$ photoproduction as a function of $|t|$ found using the Buchmüller-Tye potential as well as the BK and bSat models for $W=100 \mathrm{GeV}$ (left panel) and $W=55 \mathrm{GeV}$ (right panel). The $\psi(1 S)$ results are compared to the corresponding data from the H1 Collaboration [53,54].

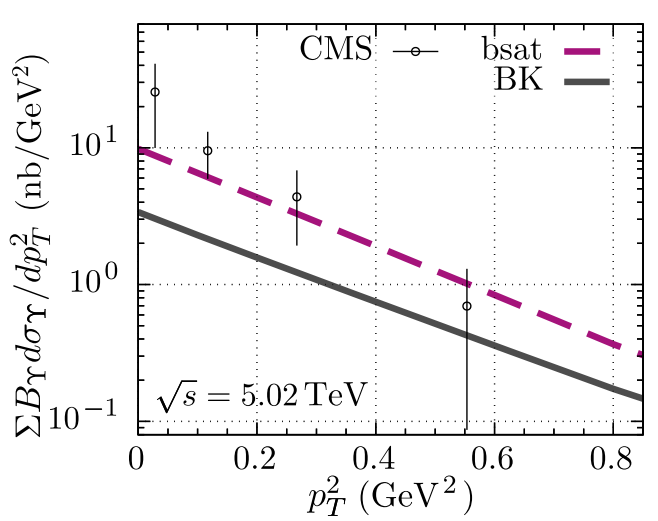

FIG. 6. Differential cross section for the $p P b \rightarrow \Upsilon(n S) p P b$ process via $\gamma p \rightarrow \Upsilon(n S) p$ as a function of $p_{T}^{2}$ calculated with the Buchmüller-Tye potential. The results using the BK and bSat dipole amplitudes are compared to the CMS data [14].

contribution multiplied by its branching fraction in the dimuon decay channel $B_{\Upsilon(n S)}$, which is taken from Ref. [55]. We notice that the models underestimate the $\Upsilon$ photoproduction data at small $p_{T}^{2}$ while getting closer to the data points at larger $p_{T}^{2}$. This shows that a fully satisfactory description of the data using the dipole model with an impact-parameter dependence, particularly with large impact parameters, is still missing from the literature.

\section{COHERENT PHOTOPRODUCTION OFF NUCLEAR TARGETS}

In photon-nucleus scattering, the differential cross section for coherent quarkonia $V$ photoproduction $\gamma A \rightarrow V A$ off a nuclear target with atomic mass $A$ can be found as follows,

$$
\frac{d \sigma^{\gamma A \rightarrow V A}}{d t}=\frac{1}{16 \pi}\left|\left\langle\mathcal{A}^{\gamma A}\left(x, \Delta_{T}\right)\right\rangle_{N}\right|^{2},
$$

in terms of the averaged amplitude [56]

$$
\begin{aligned}
\left\langle\mathcal{A}^{\gamma A}\right\rangle_{N}= & 2 i \int \mathrm{d}^{2} \mathbf{r} \int_{0}^{1} \mathrm{~d} z \\
& \times \int \mathrm{d}^{2} \mathbf{b} e^{-i[\mathbf{b}-(1-z) \mathbf{r}] \cdot \Delta} \Sigma_{T}\left\langle N_{A}(x, \boldsymbol{r}, \boldsymbol{b})\right\rangle_{N},
\end{aligned}
$$

where $\Sigma_{T}=\Sigma^{(1)}+\Sigma^{(2)} \partial / \partial r$, with the coefficients given in Eq. (2.9). Following Ref. [48], the dipole-nucleus scattering amplitude averaged over all possible configurations of the nucleons in the target nucleus reads

$$
\left\langle N_{A}(x, \boldsymbol{r}, \boldsymbol{b})\right\rangle_{N}=1-\left(1-\frac{T_{A}(b) \sigma_{q \bar{q}}(x, r)}{2 A}\right)^{A} .
$$

This equation was obtained using a $b$-dependent dipole amplitude parametrization in the same way as above. It differs from another approach found in Ref. [57], where a Gaussian shape was assumed to describe such $b$ dependence. The functions that appear in Eq. (5.3) are the usual (integrated) dipole cross section off the proton target, $\sigma_{q \bar{q}}(x, r)$, found in Eq. (2.6) and

$$
T_{A}(b)=\int_{-\infty}^{+\infty} \mathrm{d} z \rho_{A}(b, z), \quad \frac{1}{A} \int \mathrm{d}^{2} b T_{A}(b)=1,
$$

i.e., the thickness function of the nucleus, given in terms of the normalized Woods-Saxon distribution [58],

$$
\rho_{A}(b, z)=\frac{\mathcal{N}}{1+\exp \left[\frac{r(b, z)-c}{\delta}\right]}, \quad r(b, z)=\sqrt{b^{2}+z^{2}} .
$$

Here, $r(b, z)$ is the distance from the center of the nucleus and $\mathcal{N}$ is an appropriate normalization factor. In this work, we consider UPCs of lead nuclei, where $A=208$ and the parameters $c=6.62 \mathrm{fm}$ and $\delta=0.546 \mathrm{fm}$ are used [59].

The amplitude in Eq. (5.2) takes into account the imaginary part of the amplitude only. To incorporate the real part, one performs the following substitution, 


$$
\begin{aligned}
\sigma_{q \bar{q}}(x, r) & \Rightarrow \sigma_{q \bar{q}}(x, r)\left(1-i \frac{\pi \lambda}{2}\right), \quad \text { with } \\
\lambda & =\frac{\partial \ln \sigma_{q \bar{q}}(x, r)}{\partial \ln (1 / x)},
\end{aligned}
$$

which is analogous to the one made for the proton target case in Eq. (2.7). Furthermore, in order to introduce a skewness correction to the associated nuclear gluon density, one can multiply the dipole cross section by the corresponding skewness factor found in Eq. (2.8) as

$$
\sigma_{q \bar{q}}(r, x) \rightarrow \sigma_{q \bar{q}}(r, x) R_{g}(\lambda) .
$$

To study the rapidity distribution of the vector mesons produced in $A A$ UPCs, one needs to incorporate the incoming photon flux $n(\omega)$ into one of the incident nuclei such that

$$
\frac{d \sigma^{A A \rightarrow V A A}}{d y d t}=n(\omega) \frac{d \sigma^{\gamma A \rightarrow V A}}{d t}(y)+\{y \rightarrow-y\},
$$

where $\omega=\left(M_{V} / 2\right) e^{y}$ is the projectile photon energy in the center of mass (c.m.) of the colliding particles given in terms of the mass of the vector meson $M_{V}$ and its rapidity $y$. The photon flux can be written as $[60,61]$

$n(\omega)=\frac{2 Z_{A}^{2} \alpha_{\mathrm{em}}}{\pi}\left\{\xi K_{1}(\xi) K_{0}(\xi)-\frac{\xi^{2}}{2}\left[K_{1}^{2}(\xi)-K_{0}^{2}(\xi)\right]\right\}$,

where $K_{0,1}$ are the modified Bessel functions of the second kind, $Z_{A}$ is the charge of the projectile nucleus sourcing the photon flux, $\xi=2 \omega R_{A} / \gamma, R_{A}$ is the radius of the nucleus (in the numerical analysis below we use the $R_{A}$ value from Ref. [62]), $\gamma=\sqrt{s} / 2 m_{p}$ is the Lorentz factor, and $m_{p}$ is the proton mass.

This photon flux is also used in calculations of the cross section as a function of transverse momenta in $p A$ UPCs. The latter is computed in a similar way as Eq. (5.8), except that we multiply the $\gamma p$ differential cross section by the photon flux (5.9) in the first term, whereas the $\gamma A$ differential cross section is multiplied by the proton flux in the second term. At very small $t$ the $\gamma A$ (photon from proton) contribution is relevant; however, for not so small $t$, it falls much quicker than the $\gamma p$ (photon from nucleus) contribution, and therefore this second term can be disregarded safely.

The gluon density inside a nucleus at small $x$ is expected to be suppressed relative to the one inside a free nucleon caused by a relative reduction of the dipole cross section due to interference between incoming dipoles in the presence of the higher Fock states of the photon (see, e.g., Refs. [26,56,63] for more details). This phenomenon, also known as nuclear (or gluon) shadowing, effectively reduces the quarkonia photoproduction $\sigma^{\gamma A \rightarrow V A}$ cross section off a heavy nuclear target in comparison to that off the proton, $A \sigma^{\gamma p \rightarrow V p}$. Such a shadowing effect plays the most important role at central rapidities of the meson, and it can be phenomenologically incorporated by "renormalizing" the dipole cross section as

$$
\sigma_{q \bar{q}}(x, r) \rightarrow \sigma_{q \bar{q}}(x, r) R_{G}\left(x, \mu^{2}\right),
$$

where $R_{G}$ is given in terms of a ratio of the gluon density function inside the heavy nucleus $x g_{A}\left(x, \mu^{2}\right)$ over the one inside the proton $x g_{p}\left(x, \mu^{2}\right)$ as

$$
R_{G}\left(x, \mu^{2}\right)=\frac{x g_{A}\left(x, \mu^{2}\right)}{A x g_{p}\left(x, \mu^{2}\right)} .
$$

There is still a large uncertainty in the determination of the $R_{G}$ factor, and we highlight the past work of Refs. [64,65] for a thorough discussion of this issue. In our calculations, we employ the EPPS16 parametrization for the nuclear gluon distribution fitted to the LHC data [66], adopting $\mu=M_{V} / 2$ as the factorization scale [67]. As discussed in our previous paper [33], the EPPS16 parametrization was one that has provided a more satisfactory description of the data using the standard factorization scale; this fact motivates us to repeat this choice.

Besides the nuclear shadowing effect, there is another important correction to the coherent photoproduction cross section off a nucleus that is worth mentioning. To obtain the above equations, we used the Glauber-Gribov approach, which takes into account the fact that the inelastic interactions with the nucleons in the target nucleus can produce particles that shortly thereafter can be absorbed by another bound nucleon, thereby effectively making the nucleus more transparent. These inelastic corrections are calculated considering that at high energies the dipole is an eigenstate of interaction, with its transverse separation being "frozen" in the course of its propagation through the target nucleus $[68,69]$. This is called the frozen approximation and guarantees that there will be no fluctuations of the $q \bar{q}$ dipole inside the nucleus. This approximation is only valid if the lifetime of the $q \bar{q}$ state, or the so-called coherence length,

$$
l_{c}=\frac{2 \nu}{M_{V}^{2}},
$$

is much larger than the nuclear radius, i.e., $l_{c} \gg R_{A}$. Here, $\nu$ is the energy of the photon in the nucleus rest frame.

In the case where the coherence length is finite [i.e., when it is not much larger than the nuclear radius $\left(l_{c} \lesssim R_{A}\right)$ ], one needs to incorporate additional corrections into the differential cross section $d \sigma^{\gamma A \rightarrow V A} / d t$ which depend on the c.m. energy $W$. This effect occurs because the photon can propagate through the nucleus without experiencing any attenuation until the $Q \bar{Q}$ fluctuation is produced. This propagation through the nucleus can be 

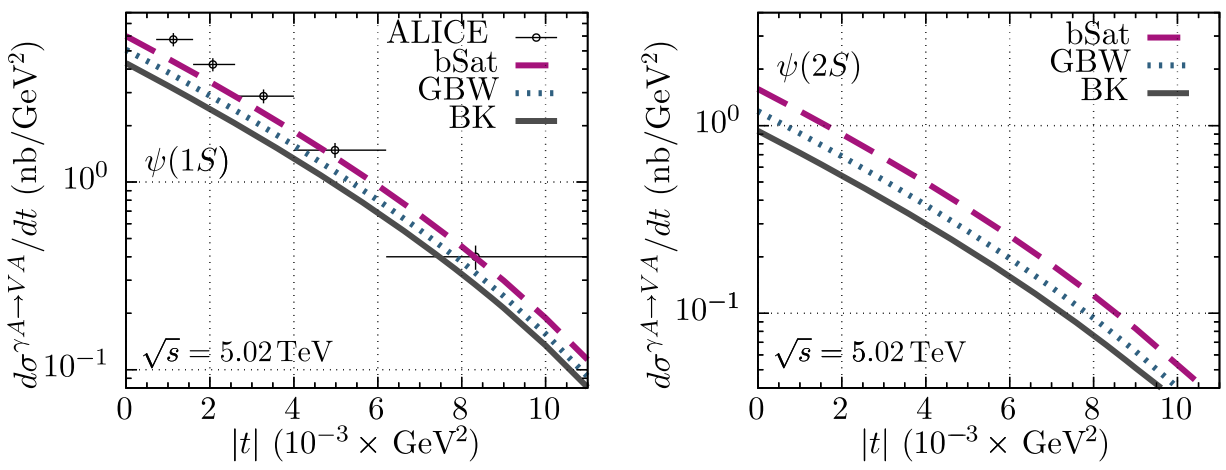

FIG. 7. Differential cross sections for the $\gamma P b \rightarrow \psi(n S) P b$ process as functions of $|t|$, with wave functions calculated using the Buchmüller-Tye potential. The results using the BK and bSat dipole amplitudes and the purely phenomenological GBW dipole cross section [73] are compared to the recent ALICE data [12] for $\psi(1 S)$.

described mathematically by a light-cone Green's function that satisfies a two-dimensional equation of motion (for more details, see Ref. [70]), whose solution is known only for the quadratic dependence of the dipole cross section approximation $\sigma_{q \bar{q}} \propto r^{2}$ and for the oscillator form of the interquark potential. In our previous work [33], we used a simplified way to take this effect into account by multiplying the nuclear cross sections in the infinite coherence length limit by a form factor that can be found in Ref. [56]. It was shown in a recent work [71] that such an estimate is valid with a reasonable accuracy only for photoproduction of $\rho$ mesons. Thus, Kopeliovich et al. compared the vector dominance model to the approach based on the light-cone Green's function and showed that there is a substa ntial difference between the form factors computed within each approach for small values of energy, mainly for the incoherent case.

The effect of the finite coherence length is known to be sizable only at large values of rapidity, where there have not been many measured data points. In this work we are focused on making the predictions for differential quarkonia photoproduction cross sections at the LHC energies, and we choose to evaluate all the results for $y=0$. At this value, the finite coherence length effect does not affect the cross section, so it can be safely disregarded.

\section{RESULTS FOR $\gamma A$ AND $A A$ COLLISIONS}

We would now like to present the numerical results for the differential cross sections of coherent vector-meson production in $\gamma A$ collisions as well as in $A A$ UPCs. As was described above, in our numerical calculations we employ the potential approach for $1 S$ and $2 S$ charmonia and bottomonia LF wave functions incorporating the Melosh spin rotation. We choose to show the results obtained with the Buchmüller-Tye potential since the difference from other potentials does not appear to be significant for this analysis. The nuclear target dipole cross section will be built on top of three dipole-proton cross sections: the numerical solution of the BK equation, the phenomenological Golec-Biernat-Wüstoff (GBW) model, and the bSat model. Furthermore, the effects of the nuclear shadowing have been accounted for by using a phenomenological approach fitted to the data [66].

In an attempt to improve the color dipole models available in the literature, Kopeliovich et al. [72] included a correlation between the impact parameter and the dipole separation in the elastic dipole-proton amplitude by adding a dependence on the angle between $\vec{r}$ and $\vec{b}$ to the color dipole cross section. It is indeed known that the interaction is amplified when $\vec{r}$ is parallel to $\vec{b}$ and vanishes when $\vec{r} \perp \vec{b}$. However, since there is an integration over all possible angles in the transverse plane, the inclusion of this correlation is not expected to have a significant impact on the final results presented in this paper.

In Fig. 7 we compare the results for the differential cross section of the $\psi(1 S)$ state to the recent ALICE data [12] at $\sqrt{s}=5.02 \mathrm{TeV}$. The models underestimate the $\psi(1 S)$ data for photoproduction at small $|t|$, while they get closer to the available data at larger $|t|$. We also provide predictions for the differential cross section of $\psi(2 S)$ at the same energy.

Predictions are presented in Fig. 8 for the differential cross section of the $\gamma P b \rightarrow V P b$ coherent photoproduction of $\psi$ states (left panel) and $\Upsilon$ states (right panel) at $\sqrt{s}=$ $5.02 \mathrm{TeV}$ for a large range of $|t|$. We notice in the figure that the positions of the dips are almost the same for both $\psi(1 S, 2 S)$ and $\Upsilon(1 S, 2 S)$, which is due to the destructive interference of individual scattering amplitudes of the nucleons of the target nucleus.

In Fig. 9 we present a similar plot but for the $A A \rightarrow V A A$ process in $A A$ UPCs for the LHC conditions (with lead nuclei), namely, at $\sqrt{s}=5.02 \mathrm{TeV}$. We also choose central $(y=0)$ rapidity in order to maximize the corresponding differential cross sections, and hence to increase the possibility of detection at the LHC. One obvious thing to mention is that these results have exactly the same shape as the ones in Fig. 8, except that they are 3 orders of 

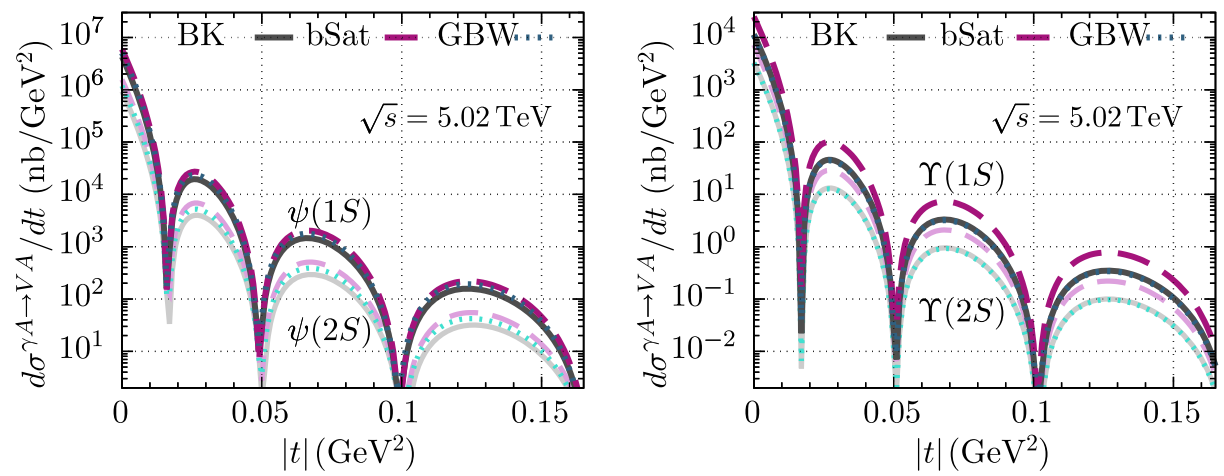

FIG. 8. Predictions for the differential cross sections for the $\gamma P b \rightarrow V P b$ processes as functions of $|t|$, calculated with three dipole cross section models: the numerical solution of the BK equation for the dipole amplitude, the bSat model, and the GBW parametrization. The results for the production of $\psi$ states (left panel) and $\Upsilon$ states (right panel) are shown. Both panels present the results at $y=0$ and with $\sqrt{s}=5.02 \mathrm{TeV}$.
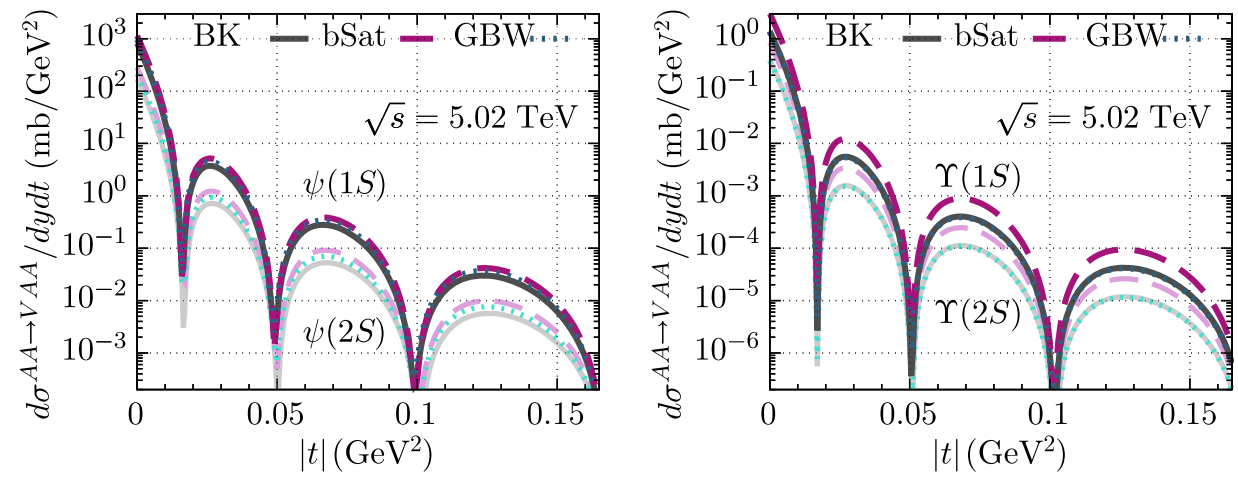

FIG. 9. Predictions for the differential cross sections for $P b P b \rightarrow V P b P b$ processes as a function of $|t|$. We show the production of $\psi$ states (left panel) and $\Upsilon$ states (right panel) at $\sqrt{s}=5.02 \mathrm{TeV}$ and $y=0$.

magnitude larger due to the photon flux. We have checked the validity of using the BK equation for a single nucleon plus the Glauber-Gribov approach together with some gluon shadowing by comparing our results to those given by Bendova et al. [74], who used the BK equation for the entire nucleus and found good agreement.

\section{CONCLUSIONS}

In this work, the impact-parameter $b$-dependent dipole model has been employed for studies of differential (in momentum transfer squared $t$ ) observables of elastic (coherent) quarkonia photoproduction off proton and nuclear targets. In the treatment of quarkonia light-front wave functions, our work has relied on the potential approach. Here, a radial-wave solution of the Schrödinger equation for a given interquark potential has first been obtained in the $Q \bar{Q}$ rest frame and then boosted to the infinite momentum frame while the spin-dependent part of the wave function has been computed by means of the Melosh transformation. We have also incorporated the skewness effect into the partial dipole amplitude at the $\gamma p$ level, while in the nuclear case the dipole cross section for an elementary dipole scattering off a single nucleon has been multiplied by such a correction factor-not the entire $\gamma A$ amplitude. In addition, the gluon shadowing effect in photoproduction off a heavy nucleus target has been accounted for fully phenomenologically.

The use of the Buchmüller-Tye potential together with the $b$-dependent solution of the BK equation for the dipoletarget amplitude has enabled us to reproduce the $\mathrm{H} 1$ data available from the HERA collider for the differential $J / \psi$ photoproduction cross section with the proton target well. The same setup has been used to make predictions for the $t$ dependent photoproduction $\gamma p \rightarrow V p$ cross section of $\psi(2 S), \Upsilon(1 S)$, and $\Upsilon(2 S)$ vector mesons. A comparison with the corresponding results obtained by using the bSat model has revealed that the latter model gives a slightly larger cross section. This is the first calculation using a realistic potential model for the excited-state wave functions and based on the latest developments in the $b$ dependent BK equation described above.

Furthermore, new predictions for the differential $\gamma P b \rightarrow$ $V P b$ and $P b P b \rightarrow V P b P b$ cross sections at central rapidity have been reported for both ground and excited $\psi$ and $\Upsilon$ 
states. Our calculations are based upon the Glauber-Gribov picture of high energy scattering and have included the gluon shadowing from a recent parametrization of nuclear PDFs. A single framework consistently combining these important elements has not yet been developed in the literature. The new data on the $J / \psi$ meson photoproduction recently published by the ALICE Collaboration made it possible to test our approach. In this case, the bSat model provides a better description of the data than the other models, showing that there are still significant uncertainties in the modeling of the $b$-dependent color dipole cross section.

Finally, the results on the differential cross section for $\Upsilon(n S)$ photoproduction via $\gamma p$ interaction in $p P b$ collisions have been compared with the existing CMS data, and, again, the bSat model predictions appear to be closer to the data points than those of the BK model. Ultimately, these calculations are expected to be of great importance for further deeper investigations of the quarkonia coherent photoproduction mechanisms in ultraperipheral collisions in the future measurements at the LHC and at the electronion collider [75].

\section{ACKNOWLEDGMENTS}

This work was supported by Fapesc, INCT-FNA (464898/2014-5), and CNPq (Brazil) for C. H., E. G. d. O., and H. T. This study was financed in part by the Coordenação de Aperfeiçoamento de Pessoal de Nível Superior-Brasil (CAPES)-Finance Code No. 001. The work has been performed in the framework of COST Action No. CA15213 "Theory of Hot Matter and Relativistic Heavy-Ion Collisions" (THOR). R. P. is supported in part by Swedish Research Council Contracts No. 621-2013-4287 and No. 2016-05996, as well as by the European Research Council (ERC) under the European Union's Horizon 2020 research and innovation program (Grant Agreement No. 668679).
[1] H. Mäntysaari, Rep. Prog. Phys. 83, 082201 (2020).

[2] S. Glazov (H1 Collaboration), Braz. J. Phys. 37, 793 (2007).

[3] W. Schäfer, Eur. Phys. J. A 56, 231 (2020).

[4] R. Aaij et al. (LHCb Collaboration), J. High Energy Phys. 09 (2015) 084.

[5] A. Bursche (LHCb Collaboration), Nucl. Phys. A982, 247 (2019).

[6] R. Aaij et al. (LHCb Collaboration), J. High Energy Phys. 10 (2018) 167.

[7] B. Abelev et al. (ALICE Collaboration), Phys. Lett. B 718, 1273 (2013).

[8] E. Abbas et al. (ALICE Collaboration), Eur. Phys. J. C 73, 2617 (2013).

[9] J. Adam et al. (ALICE Collaboration), Phys. Lett. B 751, 358 (2015).

[10] E. L. Kryshen (ALICE Collaboration), Nucl. Phys. A967, 273 (2017).

[11] S. Acharya et al. (ALICE Collaboration), Phys. Lett. B 798, 134926 (2019).

[12] S. Acharya et al. (ALICE Collaboration), Phys. Lett. B 817, 136280 (2021).

[13] V. Khachatryan et al. (CMS Collaboration), Phys. Lett. B 772, 489 (2017).

[14] A. M. Sirunyan et al. (CMS Collaboration), Eur. Phys. J. C 79, 277 (2019).

[15] H. Kowalski, L. Motyka, and G. Watt, Phys. Rev. D 74, 074016 (2006).

[16] I. Balitsky, Nucl. Phys. B463, 99 (1996).

[17] Y. V. Kovchegov, Phys. Rev. D 61, 074018 (2000).

[18] B. Ducloué, E. Iancu, G. Soyez, and D. N. Triantafyllopoulos, Phys. Lett. B 803, 135305 (2020).

[19] D. Bendova, J. Cepila, J. G. Contreras, and M. Matas, Phys. Rev. D 100, 054015 (2019).
[20] J. Cepila, J. G. Contreras, and M. Matas, Phys. Rev. C 102, 044318 (2020).

[21] S. Martins and M. B. Gay Ducati, Int. J. Mod. Phys. Conf. Ser. 45, 1760058 (2017).

[22] M. B. Gay Ducati, F. Kopp, M. V. T. Machado, and S. Martins, Proc. Sci., ICHEP2016 (2016) 363.

[23] Y. Hatta, B.-W. Xiao, and F. Yuan, Phys. Rev. Lett. 116, 202301 (2016).

[24] B. Z. Kopeliovich, L. I. Lapidus, and A. B. Zamolodchikov, Pis'ma Zh. Eksp. Teor. Fiz. 33, 612 (1981) [JETP Lett. 33, 595 (1981)], http://jetpletters.ru/ps/1511/article_23095.shtml.

[25] N. N. Nikolaev and B. G. Zakharov, Zh. Eksp. Teor. Fiz. 105, 1117 (1994) [J. Exp. Theor. Phys. 78, 598 (1994)].

[26] B. Z. Kopeliovich and B. G. Zakharov, Phys. Rev. D 44, 3466 (1991).

[27] B. Z. Kopeliovich, J. Nemchick, N. N. Nikolaev, and B. G. Zakharov, Phys. Lett. B 324, 469 (1994).

[28] J. Nemchik, N. N. Nikolaev, and B. G. Zakharov, Phys. Lett. B 341, 228 (1994).

[29] J. Nemchik, N. N. Nikolaev, E. Predazzi, and B. G. Zakharov, Z. Phys. C 75, 71 (1997).

[30] M. B. Gay Ducati, M. T. Griep, and M. V. T. Machado, Phys. Rev. C 88, 014910 (2013).

[31] J. Hufner, Yu. P. Ivanov, B. Z. Kopeliovich, and A. V. Tarasov, Phys. Rev. D 62, 094022 (2000).

[32] A. Shuvaev, K. J. Golec-Biernat, A. D. Martin, and M. Ryskin, Phys. Rev. D 60, 014015 (1999).

[33] C. Henkels, E. G. de Oliveira, R. Pasechnik, and H. Trebien, Phys. Rev. D 102, 014024 (2020).

[34] A. Martin, Phys. Lett. 93B, 338 (1980).

[35] N. Barik and S. N. Jena, Phys. Lett. 97B, 265 (1980).

[36] E. Eichten, K. Gottfried, T. Kinoshita, K. D. Lane, and T.-M. Yan, Phys. Rev. D 17, 3090 (1978); 21, 313(E) (1980). 
[37] E. Eichten, K. Gottfried, T. Kinoshita, K. D. Lane, and T.-M. Yan, Phys. Rev. D 21, 203 (1980).

[38] W. Buchmuller and S.H.H. Tye, Phys. Rev. D 24, 132 (1981).

[39] C. Quigg and J. L. Rosner, Phys. Lett. 71B, 153 (1977).

[40] H. J. Melosh, Phys. Rev. D 9, 1095 (1974).

[41] M. Krelina, J. Nemchik, R. Pasechnik, and J. Cepila, Eur. Phys. J. C 79, 154 (2019).

[42] J. Cepila, J. Nemchik, M. Krelina, and R. Pasechnik, Eur. Phys. J. C 79, 495 (2019).

[43] H. Mäntysaari and J. Penttala, arXiv:2104.02349.

[44] T. Lappi, H. Mäntysaari, and J. Penttala, Phys. Rev. D 102, 054020 (2020).

[45] B. Sambasivam, T. Toll, and T. Ullrich, Phys. Lett. B 803, 135277 (2020).

[46] V. P. Gonçalves, D. E. Martins, and C. R. Sena, Eur. Phys. J. A 57, 82 (2021).

[47] S. Dulat, T.-J. Hou, J. Gao, M. Guzzi, J. Huston, P. Nadolsky, J. Pumplin, C. Schmidt, D. Stump, and C. Yuan, Phys. Rev. D 93, 033006 (2016).

[48] H. Kowalski and D. Teaney, Phys. Rev. D 68, 114005 (2003).

[49] E. Iancu, J. Madrigal, A. Mueller, G. Soyez, and D. Triantafyllopoulos, Phys. Lett. B 750, 643 (2015).

[50] S. Drell and T.-M. Yan, Phys. Rev. Lett. 24, 181 (1970).

[51] S. J. Brodsky and G. R. Farrar, Phys. Rev. Lett. 31, 1153 (1973).

[52] G. B. West, Phys. Rev. Lett. 24, 1206 (1970).

[53] C. Alexa et al. (H1 Collaboration), Eur. Phys. J. C 73, 2466 (2013).

[54] A. Aktas et al. (H1 Collaboration), Eur. Phys. J. C 46, 585 (2006).

[55] C. Patrignani et al. (Particle Data Group), Chin. Phys. C 40, 100001 (2016).

[56] Yu. P. Ivanov, B. Z. Kopeliovich, A. V. Tarasov, and J. Hüfner, Phys. Rev. C 66, 024903 (2002).
[57] Y.-P. Xie and X. Chen, Nucl. Phys. A959, 56 (2017).

[58] R. D. Woods and D.S. Saxon, Phys. Rev. 95, 577 (1954).

[59] H. Euteneuer, J. Friedrich, and N. Vogler, Nucl. Phys. A298, 452 (1978).

[60] C. F. von Weizsacker, Z. Phys. 88, 612 (1934).

[61] E. J. Williams, Phys. Rev. 45, 729 (1934).

[62] H. De Vries, C. De Jager, and C. De Vries, At. Data Nucl. Data Tables 36, 495 (1987).

[63] Y. P. Ivanov, B. Z. Kopeliovich, A. V. Tarasov, and J. Hufner, AIP Conf. Proc. 660, 283 (2003).

[64] I. Schienbein, J. Y. Yu, K. Kovařík, C. Keppel, J. G. Morfín, F. I. Olness, and J. F. Owens, Phys. Rev. D 80, 094004 (2009).

[65] D. de Florian, R. Sassot, P. Zurita, and M. Stratmann, Phys. Rev. D 85, 074028 (2012).

[66] K. J. Eskola, P. Paakkinen, H. Paukkunen, and C. A. Salgado, Eur. Phys. J. C 77, 163 (2017).

[67] V. Guzey, E. Kryshen, and M. Zhalov, Phys. Rev. C 93, 055206 (2016).

[68] B. Kopeliovich, I. Potashnikova, and I. Schmidt, Phys. Rev. C 73, 034901 (2006).

[69] B. Kopeliovich, Int. J. Mod. Phys. A 31, 1645021 (2016).

[70] B. Kopeliovich, J. Nemchik, A. Schafer, and A. Tarasov, Phys. Rev. C 65, 035201 (2002).

[71] B. Kopeliovich, M. Krelina, J. Nemchik, and I. Potashnikova, arXiv:2008.05116.

[72] B. Z. Kopeliovich, M. Krelina, and J. Nemchik, Phys. Rev. D 103, 094027 (2021).

[73] K. J. Golec-Biernat and M. Wusthoff, Phys. Rev. D 60, 114023 (1999).

[74] D. Bendova, J. Cepila, J. Contreras, and M. Matas, Phys. Lett. B 817, 136306 (2021).

[75] A. Accardi et al., Eur. Phys. J. A 52, 268 (2016). 